\title{
The epidemiology and treatment of mesothelioma in South East England 1985-2002
}

\author{
V Mak, E Davies, V Putcha, B Choodari-Oskooei, H Møller
}

King's College London, Thames Cancer Registry, London, UK

Correspondence to: V Mak, King's College London, Thames Cancer Registry, 1st

Floor, Capital House, 42 Weston Street, London, SE1 30D, UK; vivian.mak@kcl.ac.uk

Received 21 June 2006 Accepted 20 July 2007

Published Online First

3 August 2007

\section{ABSTRACT}

Objectives: To describe trends in the incidence of mesothelioma for men and women in South East England and the geographical variation at the level of primary care trust. To describe treatment patterns by cancer network of residence, and relative survival by cancer network, disease stage and treatment modality.

Methods: 5753 cases were extracted from the Thames Cancer Registry database. We calculated age standardised incidence rates for each year, age specific incidence rates in 10 year age groups, and we used linear regression to compute the average annual percentage change in age standardised incidence. We used Poisson regression to analyse generational trends in incidence.

Results: Men had five times higher incidence of mesothelioma than women. In men, there was an overall 4\% increase per year between 1985 and 2002. Over the same period, the overall increase in incidence for women was $5 \%$ per year. The incidence was highest in men aged over 70 years, and men aged over 80 years had the highest increase of $8 \%$ per year. The incidence rate ratio increased for men born between 1892 and 1942 and started to slow for those born from 1947 onwards. Areas along the Thames and its estuary had the highest incidence. There was some variation by cancer network in the proportion of patients receiving cancer surgery, radiotherapy and chemotherapy. There were no discernable differences in relative survival by cancer network of residence or disease stage but those receiving combined treatment had higher 5 year survival.

Conclusions: Mesothelioma incidence has increased in South East England, particularly for men aged over 70 years. The highest incidence occurs along the Thames and its estuary, reflecting areas of asbestos use in shipbuilding and industry in the past. More research is needed to understand the interrelationships of prognostic factors, treatment choices and survival, and to determine the best care and support for these patients and their families.

Mesothelioma is a less common tumour with a poor prognosis. Since the 1970s, many western countries, including the UK, have observed an increasing incidence of this cancer, particularly among men. ${ }^{1}$ The link to earlier occupational exposure to asbestos, often through mining, shipbuilding or the manufacture of asbestos textiles, cement and insulation is now well established. The average latency period before the development and presentation of disease is over 30 years, and may be as long as 60 years. ${ }^{2}$ Although many countries have now banned the industrial use of asbestos, this long latency period means that new cases will continue to appear for some time. Early predictions of a peak in European mortality for the first two decades of the 21st century were based on modelling using deaths observed in cohorts of men born in the mid twentieth century. ${ }^{3}$ Some countries, including Sweden and Norway, have since reported a levelling off in the incidence rates for men, suggesting that the peak in these countries has already been reached. ${ }^{4}$ Analysis of more recent mortality data from France, Germany and Italy also suggested that the mortality peak in these countries will occur in the current decade rather than the next. ${ }^{5}$ In Great Britain, where asbestos use continued later than many other countries, the peak is anticipated to occur later between 2011 and 2115. ${ }^{6}$ Between 1981 and 2000, North East England and South East England were the areas with the highest standardised mortality ratios. $^{7}$ Asbestos related mortality in Northern Ireland is already showing a decline, with most of these deaths occurring around the shipbuilding industry areas in the Belfast estuary. ${ }^{8}$

Although public health action has undoubtedly prevented many new cases of mesothelioma, the treatment options for patients once they have developed the disease are limited. The median survival after diagnosis is less than 12 months. Surgery (extrapleural pneumonectomy) combined with postoperative radiotherapy and chemotherapy may have a role in selected patients presenting with early stage disease. ${ }^{9}$ However, for many patients the main needs will be for an accurate diagnosis, information about the condition, advice on legal implications and for high quality palliative care to help control the symptoms of pain and breathlessness, and provide family support. ${ }^{10}$ Formal evidence based national guidance on the services these patients can expect or their effectiveness has not yet been developed.

The area of South East England served by the Thames Cancer Registry includes an area of high mesothelioma incidence related to previous shipbuilding (Medway in Kent), and several others where asbestos was used previously in factories (Newham, Tower Hamlets, Barking and Dagenham, Havering and Redbridge in London, and Dartford and Gravesham in Kent). Although the number of women developing mesothelioma is five times lower than for men, factory work in these areas exposed women to asbestos, and women in South East England are among those with the highest risk in the UK.

This study set out to provide information that would guide health service planning to meet these patient needs. It aimed to

1. Describe trends in the incidence of mesothelioma for men and women in South East England between 1985 and 2002. 
2. Analyse cohort trends in incidence rate ratio of mesothelioma for men.

3. Explore the incidence of mesothelioma for men and women at the level of individual primary care trust.

4. Describe treatment patterns by cancer network of residence, and relative survival by cancer network, disease stage and treatment modality.

\section{METHOD}

The Thames Cancer Registry (TCR) covered the areas of Essex, Hertfordshire, Kent, Surrey, Sussex and London, and included a resident population of 14.2 million people at the time of this study. Cancer registration is triggered by multiple sources of information, including pathological diagnoses and cancer treatment within each hospital, and from death certificates received from the Office for National Statistics. Registry data collection officers collect demographic, diagnostic and treatment informa-

Table 1 Demographic and clinical characteristics of patients with mesothelioma (ICD-10 C45), diagnosed between 1998 and 2002, resident in South East England $(n=5753)$

\begin{tabular}{|c|c|}
\hline & $\begin{array}{l}\text { No of patients } \\
(\mathrm{n}(\%))\end{array}$ \\
\hline \multicolumn{2}{|l|}{ Sex } \\
\hline Men & $4767(83)$ \\
\hline Women & $986(17)$ \\
\hline \multicolumn{2}{|l|}{ Age group (y) } \\
\hline $0-49$ & $328(6)$ \\
\hline $50-59$ & $1094(19)$ \\
\hline $60-69$ & $1798(31)$ \\
\hline $70-79$ & $1800(31)$ \\
\hline $80+$ & $733(13)$ \\
\hline \multicolumn{2}{|l|}{ Cancer network of residence } \\
\hline Kent and Medway & $860(15)$ \\
\hline North East London & $890(15)$ \\
\hline North London & $389(7)$ \\
\hline South East London & $618(11)$ \\
\hline South Essex & $442(8)$ \\
\hline South West London & $391(7)$ \\
\hline Surrey, West Sussex and Hampshire & $484(8)$ \\
\hline Sussex & $413(7)$ \\
\hline TCR part of Central South Coast & $80(1)$ \\
\hline TCR part of Mid Anglia & $290(5)$ \\
\hline TCR part of Mount Vernon & $342(6)$ \\
\hline TCR part of West Anglia & $52(1)$ \\
\hline West London & $502(9)$ \\
\hline \multicolumn{2}{|l|}{ Mesothelioma types } \\
\hline Pleura & $5287(92)$ \\
\hline Peritoneum & $213(4)$ \\
\hline Pericardium & $3(0)$ \\
\hline Other sites & $152(3)$ \\
\hline Unspecified & $98(2)$ \\
\hline \multicolumn{2}{|l|}{ Histopathological types } \\
\hline Malignant & $5556(97)$ \\
\hline Fibrous & $54(1)$ \\
\hline Epitheloid & $106(2)$ \\
\hline Biphasic type & $37(1)$ \\
\hline \multicolumn{2}{|l|}{ TCR disease stage } \\
\hline Local & $2588(45)$ \\
\hline Extension beyond organ of origin & $353(6)$ \\
\hline Regional lymph node involvement & $45(1)$ \\
\hline Metastasis & $581(10)$ \\
\hline Not known & $2186(38)$ \\
\hline
\end{tabular}

TCR, Thames Cancer Registry. tion from the medical records in each hospital. Data are checked in the registry to ensure that they refer to a new tumour rather than a recurrence of an already registered case. Information from death certificates of patients on the cancer register allows the calculation of survival after diagnosis. TCR is dependent on the staging data recorded in the clinical notes. As this information is often not complete, it employs an additional simple staging system using all available information. This classifies solid tumours at diagnosis as "local" (stage 1), "extension beyond organ of origin" (stage 2), "regional lymph node involvement" (stage 3) and "metastasis" (stage 4). Patients whose disease stage was not determined because of lack of information were classified as having disease stage "not known".

We extracted data on 5753 incident cases of mesothelioma diagnosed between 1985 and 2002 from the registry database. We tabulated the demographic and clinical characteristics of these patients. We first calculated the age standardised incidence rate for men and women for the whole area. We used data on the size of the resident population to determine the age specific incidence rates for mesothelioma in each year for each of the groups 50-59, 60-69, 70-79 and 80+ and then for individual networks using the European standard population. We used linear regression to quantify the annual percentage change in the incidence of mesothelioma.

We used Poisson regression to perform an age cohort model to analyse generational trends in the incidence of mesothelioma in men. We compared the incidence of different generations to those born in 1927 and expressed this as the incidence rate ratio. We have analysed the cohort trends in the incidence of mesothelioma in women but decided not to include the results as it could potentially be misleading because of the small numbers of cases. We then considered variation in incidence across cancer networks within South East England for men and women.

As cancer networks cover a relatively large geographical area over which disease burden is not evenly distributed, we also

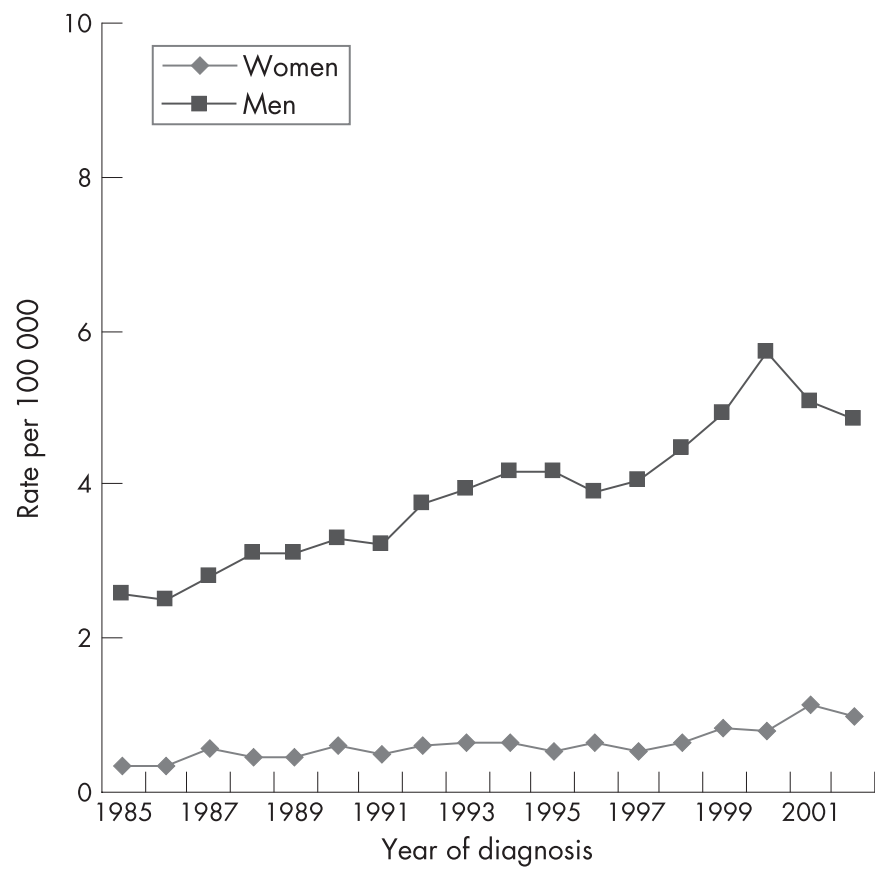

Figure 1 Age standardised incidence rates of mesothelioma, South East England, 1985-2002. 
Figure 2 Age specific incidence rates of mesothelioma, South East England, 1985-2002 in (A) men and (B) women.
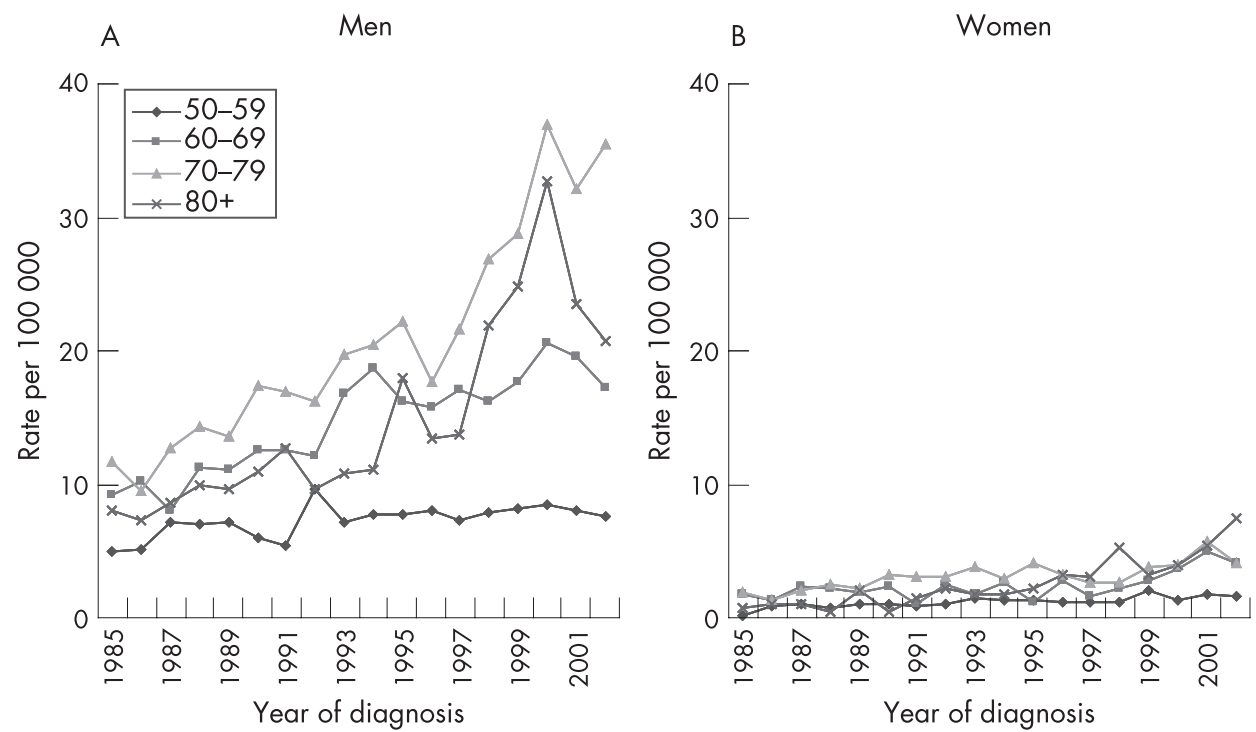

explored the variation in incidence across primary care trusts. We computed the standardised incidence ratios (SIR) using the incidence rate for each primary care trust and that for South East England as the comparator for men and women. These SIR estimates were mapped using a quartiles mapping technique. ${ }^{11}$ The primary care trusts were ranked according to their SIRs and classified into four groups. Areas with high SIRs were classified as having a high incidence of mesothelioma. These areas were shaded darker than areas with low incidence.

To explore whether treatment varied by cancer network we tabulated the proportion of patients receiving cancer surgery, other surgery, investigative surgery, radiotherapy and

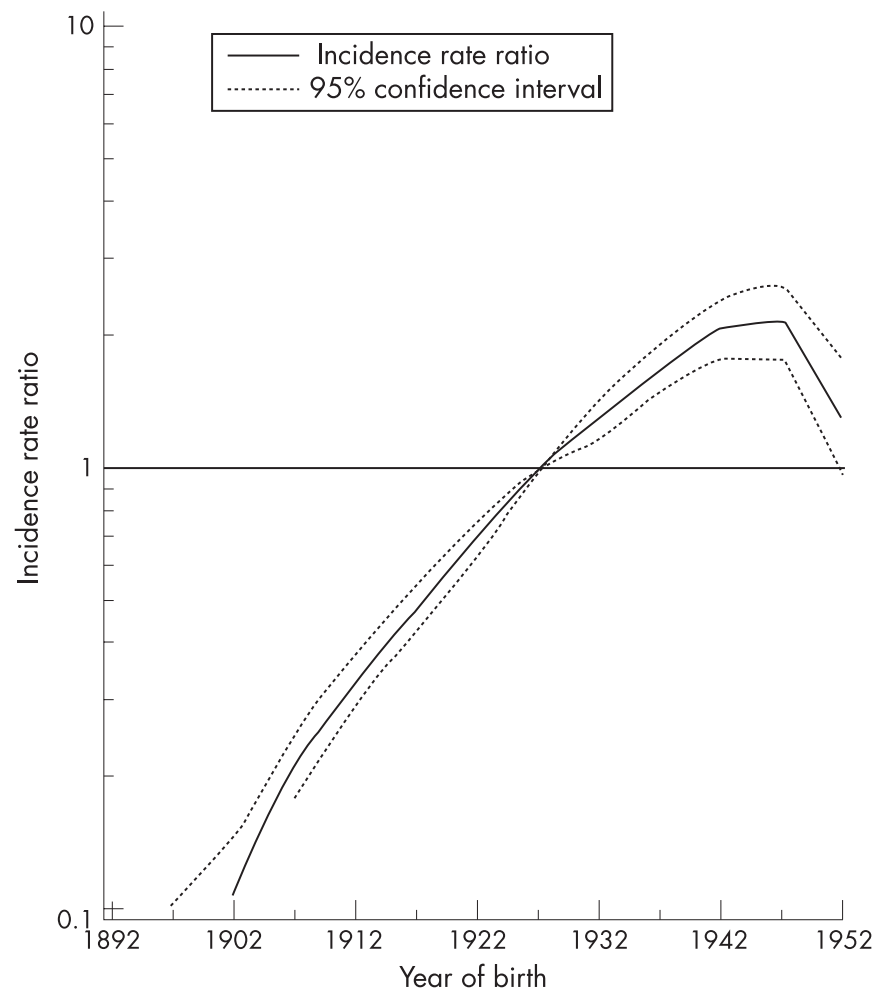

Figure 3 Cohort trends for mesothelioma incidence in men using an age cohort model, aged 25-85 years, South East England. chemotherapy, received within 6 months of diagnosis. We also analysed the proportion of patients receiving cancer surgery combined with other treatments. To determine whether survival varied among cancer networks, we used period analysis $^{12}$ to calculate relative survival for men and women with mesothelioma in the whole area. The period analysis method enables the data to be analysed cross sectionally instead of longitudinally and the computation is based on a recent period of follow-up. This enables the most recent data to be included in the computation. This analysis included only those patients for whom the period of follow-up was between 1998 and 2002 and excluded patients whose notifications were from death certificates only. We compared the relative survival of those in high and low incidence cancer networks, and for men by different disease stage and treatment modality. Because of the low number of patients in some of these categories, men with mesothelioma were further classified into those with "localised", "non-localised" (stages 2,3 and 4) and "unknown" disease stage for survival analysis. We did not calculate the relative survival for women by disease stage and treatment modality because of the low number of patients.

\section{RESULTS}

Table 1 shows the demographics and clinical characteristics of patients with mesothelioma diagnosed between 1998 and 2002 in South East England. Eighty-three per cent of the patients were men and $62 \%$ were aged between 60 and 79 years. Ninetytwo per cent were diagnosed with mesothelioma of the pleura, $97 \%$ were classified histopathologically as malignant and $45 \%$ were staged with "local" disease at diagnosis.

Figure 1 shows the age standardised incidence rates of mesothelioma for men and women in South East England. The incidence for men increased over the study period, with a sharp increase since 1996, peaking in 1999. Overall, there was a $4 \%$ annual percentage increase in the age standardised incidence rate for South East England men between 1985 and 2002. The incidence for women remained relatively stable up until 1997 followed by a slight increase thereafter.

Figure $2 \mathrm{~A}$ shows the age specific incidence rates for men in different age groups. The highest rates were found in men aged 70-79 years, followed since 1997 by those aged over 80 years. Men aged between 50 and 59 years consistently had the lowest 
Figure 4 Age standardised incidence rates of mesothelioma in men and women by cancer network, South East England, 1998-2002.

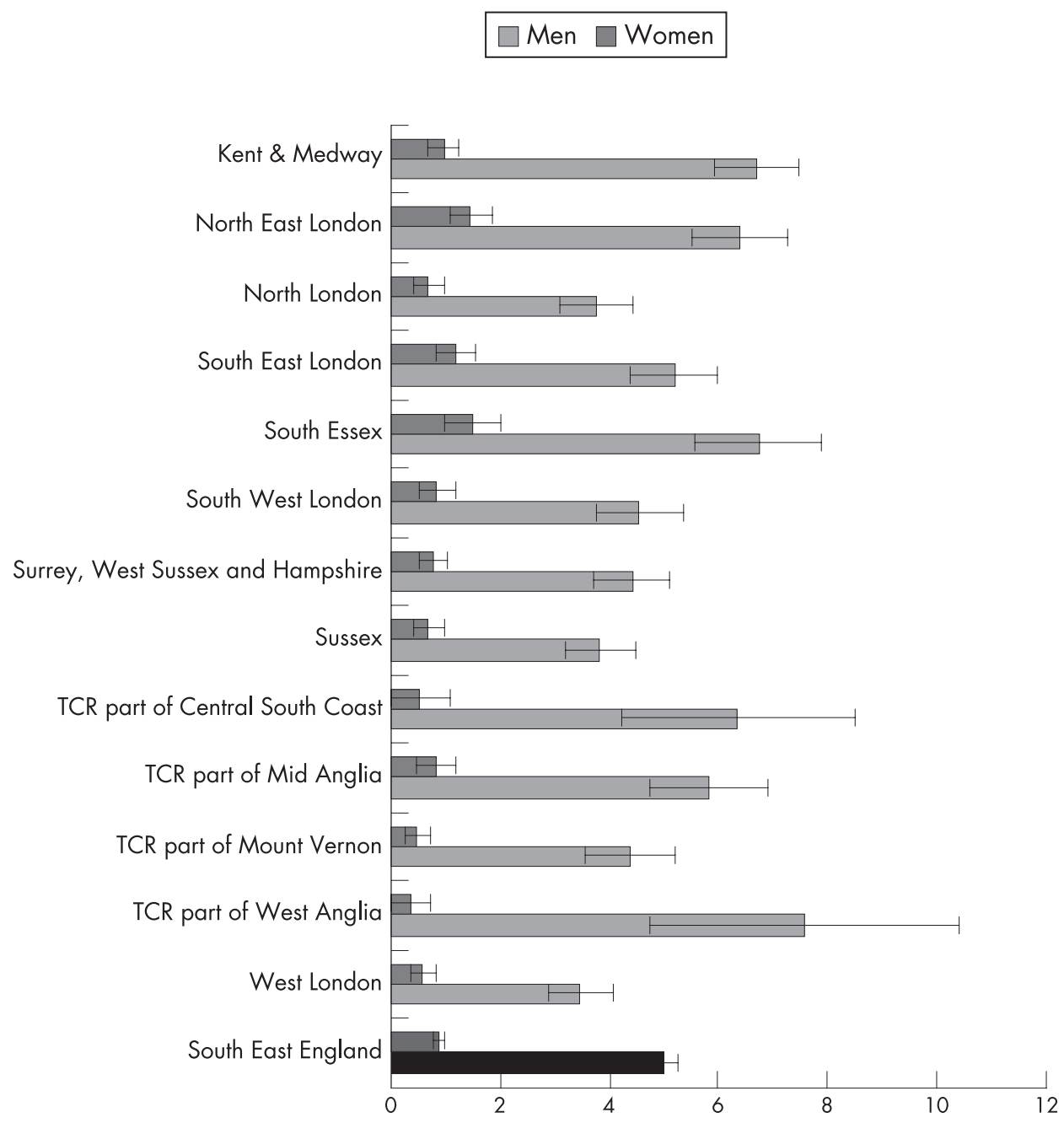

rates over the study period. The highest annual percentage increase in incidence was found in men over 80 years old (7.7\%). By contrast, the annual percentage increase in men aged between 50 and 59 years was $2.2 \%$. Figure $2 \mathrm{~B}$ shows the age specific incidence rates for women over 50 years of age. Between 1985 and 1996, the highest rates were found in women aged 70 79 years. Since 1997, women over 80 years predominantly had the highest rates. The highest annual percentage increase in incidence was also found in women over 80 years old (13.9\%).
Women aged between 60 and 69 years had the lowest annual percentage increase of $4.8 \%$.

Figure 3 shows the cohort trends of incidence rate ratios for mesothelioma in men. The incidence rate ratios for each birth cohort were compared with the 1927 birth cohort. The incidence rate ratios of mesothelioma incidence in men increased sharply from the 1892 birth cohort until 1942. Thereafter it showed a slower increase and started decreasing from the 1947 birth cohort onwards.
Figure 5 Standardised incidence ratios (SIR) of mesothelioma incidence in men and women, in each primary care trust, South East England, 1985-2002. (A) Men, (B) women.
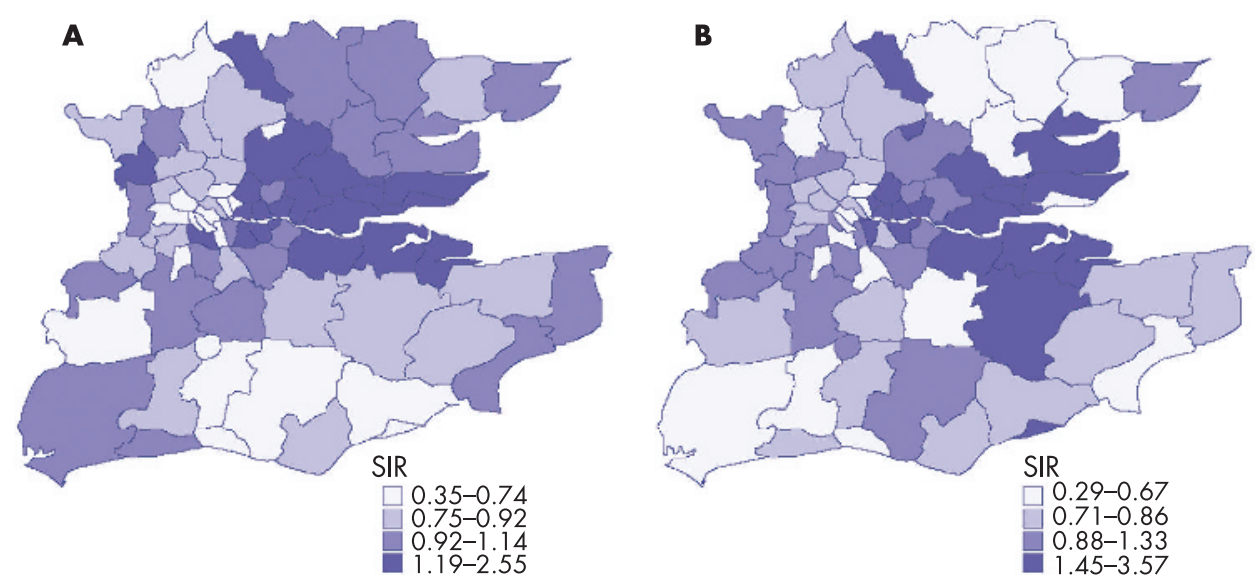
Table 2 Number of patients diagnosed with mesothelioma in each cancer network in South East England, 1998-2002, and the percentage receiving different treatments (men and women combined, $n=2188$ )

\begin{tabular}{|c|c|c|c|c|c|c|c|c|c|}
\hline Cancer network of residence & $\begin{array}{l}\text { Mean } \\
\text { No of } \\
\text { patients } \\
\text { per year }\end{array}$ & $\begin{array}{l}\% \text { Any } \\
\text { surgery }\end{array}$ & $\begin{array}{l}\% \text { Cancer } \\
\text { surgery }\end{array}$ & $\begin{array}{l}\% \text { Other } \\
\text { surgery }\end{array}$ & $\begin{array}{l}\% \\
\text { Investigative } \\
\text { surgery }\end{array}$ & $\%$ Radiotherapy & \% Chemotherapy & $\begin{array}{l}\% \text { No } \\
\text { treatment } \\
\text { recorded }\end{array}$ & $\begin{array}{l}\% \text { Death } \\
\text { certificate } \\
\text { only }\end{array}$ \\
\hline Kent \& Medway & 72 & 75 & 61 & 7 & 7 & 31 & 24 & 15 & 4 \\
\hline North East London & 54 & 82 & 57 & 11 & 13 & 25 & 19 & 10 & 4 \\
\hline North London & 29 & 75 & 50 & 12 & 14 & 31 & 22 & 13 & 2 \\
\hline South East London & 44 & 72 & 56 & 9 & 8 & 34 & 16 & 18 & 3 \\
\hline South Essex & 34 & 77 & 56 & 4 & 18 & 40 & 11 & 15 & 2 \\
\hline South West London & 31 & 74 & 59 & 7 & 8 & 37 & 32 & 10 & 4 \\
\hline Surrey, West Sussex \& Hampshire & 39 & 78 & 59 & 4 & 15 & 39 & 23 & 9 & 3 \\
\hline Sussex & 34 & 76 & 51 & 8 & 17 & 30 & 9 & 15 & 4 \\
\hline TCR part of Central South Coast & 8 & 74 & 50 & 2 & 17 & 14 & 14 & 19 & 5 \\
\hline TCR part of Mid Anglia & 27 & 80 & 62 & 5 & 13 & 31 & 12 & 10 & 4 \\
\hline TCR part of Mount Vernon & 26 & 78 & 48 & 20 & 10 & 22 & 10 & 15 & 4 \\
\hline TCR part of West Anglia & 6 & 60 & 47 & 7 & 7 & 30 & 23 & 20 & 3 \\
\hline West London & 33 & 71 & 37 & 18 & 16 & 26 & 14 & 15 & 7 \\
\hline South East England & 438 & 76 & 55 & 9 & 12 & 31 & 18 & 14 & 4 \\
\hline
\end{tabular}

Figure 4 shows the age standardised incidence rates by cancer network. The South Essex, Kent and Medway, and North East London cancer networks had higher incidence rates for men and women.

Figure 5 shows the variation in standardised incidence ratios for mesothelioma in men (fig 5A) and women (fig 5B) across the primary care trusts in South East England. Primary care trusts along the Thames and its estuary had the highest incidence, extending from the City and Hackney in north east London to Maldon and South Chelmsford in Essex, and from Greenwich in south east London to Medway and Swale in Kent.

Table 2 shows the average number of cases in each cancer network and the percentage of patients who received cancer surgery, other surgery, investigative surgery, radiotherapy and chemotherapy. Overall, 76\% (332/438) had surgery, 31\% (136/ 438) had radiotherapy and 18\% (81/438) had chemotherapy. There was some variation between cancer networks in the treatment that patients received. Considering only the nine cancer networks completely covered by the TCR, 37-61\% of patients received some form of surgery for their cancer, between $25 \%$ and $40 \%$ of patients received radiotherapy and $9-32 \%$ received chemotherapy. Overall, 14\% of patients did not have any treatment recorded. But the proportion of elderly patients aged over 80 years with no treatment recorded was high at $24 \%$.

Table 3 shows the combined treatment modalities of mesothelioma patients recorded as having received cancer surgery, resident in South East England, diagnosed between

Table 3 Patients diagnosed with mesothelioma in South East England 1998-2002 who received cancer surgery combined with other treatment modalities (men and women combined, $\mathrm{n}=1199$ )

\begin{tabular}{lll}
\hline Treatment modality & $\begin{array}{l}\text { Mean } \\
\text { No of } \\
\text { patients } \\
\text { per year }\end{array}$ & $\begin{array}{l}\% \text { of } \\
\text { patients }\end{array}$ \\
\hline Surgery only & 125 & 52 \\
Surgery combined with radiotherapy & 65 & 27 \\
Surgery combined with chemotherapy & 28 & 12 \\
Surgery combined with radiotherapy and chemotherapy & 21 & 9 \\
Surgery combined with either radiotherapy or chemotherapy & 2 & 1 \\
$\quad$ and/or hormone therapy & 240 & - \\
Total & & \\
\hline
\end{tabular}

1998 and 2002. There were 1199 patients in total and 52\% (623/ $1199)$ received surgery alone, $27 \%$ (323/1199) received surgery combined with radiotherapy and 12\% (138/1199) received surgery combined with chemotherapy.

Figure 6 shows the 5 year relative survival in men (fig 6A) and women (fig 6B) in South East England. There was no difference in survival between patients from cancer networks with high and low incidence. The 1 year and 5 year relative survival for men in South East England was 28\% and 3\%, respectively. The corresponding relative survival for women was $36 \%$ and $6 \%$.

Figure 7 shows the 5 year relative survival of men with mesothelioma by disease stage. The 1 year and 5 year relative survival for men with localised disease was $33.1 \%$ and $2.9 \%$, respectively. The corresponding relative survival for men with non-localised disease stage was $16.6 \%$ and $1.2 \%$. The 1 year and 5 year relative survival for men with unknown disease stage was $21.8 \%$ and $2.8 \%$, respectively.

Figure 8 shows the 5 year relative survival of men with mesothelioma by treatment type. The 1 year and 5 year relative survival for men who received surgery alone was $21.8 \%$ and $2.6 \%$, respectively. Those who received surgery and radiotherapy had 1 and 5 year relative survival of $41.0 \%$ and $2.2 \%$. The 1 year and 5 year relative survival for men who were treated with surgery and chemotherapy was $43.7 \%$ and $4.3 \%$, respectively. The corresponding relative survival for men with other combined treatments was $27.8 \%$ and $3.9 \%$, respectively. The 1 year and 5 year relative survival for men with no treatment recorded was $17.8 \%$ and $1.8 \%$, respectively.

\section{DISCUSSION}

\section{Summary of main findings}

In this study, we found that the incidence of mesothelioma in the population of South East England was five times higher in men than in women, and increased by 4\% per year from 1985 to 2002. Men aged between 70 and 79 years had the highest incidence and men aged over 80 years had the highest increase in incidence of $8 \%$ per year. The birth cohort analysis showed that the incidence rate ratios in men declined in successive generations born after about 1947. The results confirm the long latency period for this disease which is likely to be related to occupational exposure to asbestos before the 1980s. The incidence was higher in areas covered by South Essex, Kent 
Figure 6 Relative survival of mesothelioma by cancer networks (CN) of high and low incidence, South East England (1998-2002). (A) Men, (B) women.
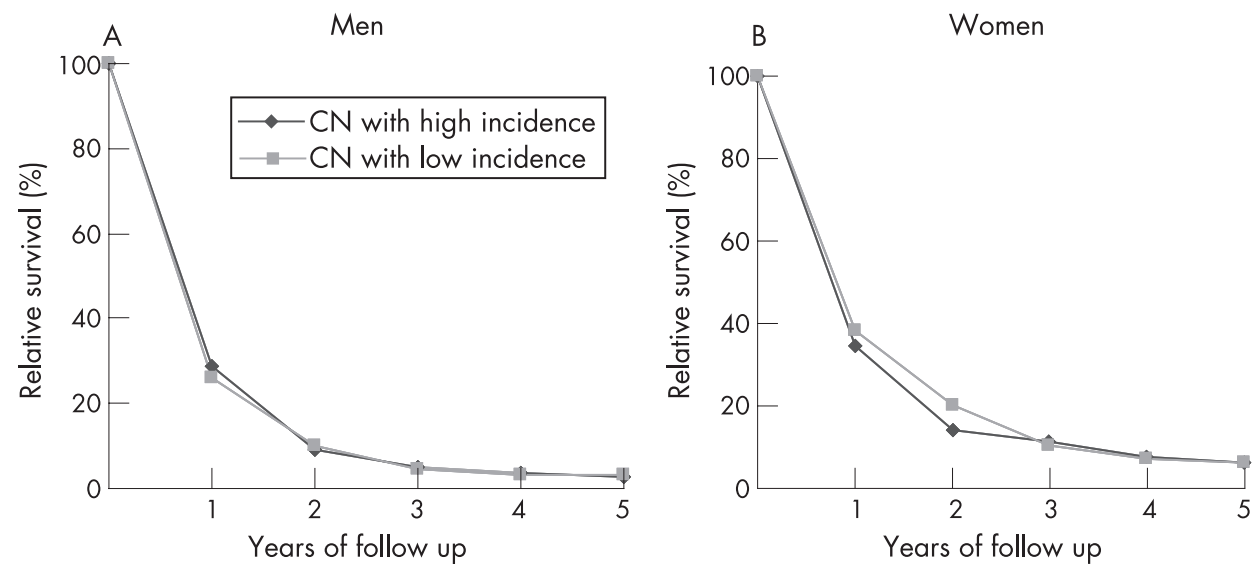

and Medway, and North East London cancer networks. These areas of highest incidence were along the Thames and its estuary, stretching from the City of London to Maldon and South Chelmsford, and from Greenwich to Medway and Swale. We also found variation in treatment given across cancer networks. Overall, considering only cancer networks completely covered by our registration area, $55 \%$ of patients received cancer surgery (range 37-61 by network); 31\% received some radiotherapy (range $25-40$ by network) and 18\% received some chemotherapy (range 9-32 by network). However, there was no difference in relative survival between patients treated in networks of high and low incidence. There was minimal difference in relative survival between men with localised and non-localised disease stage. Men who were treated with surgery and chemotherapy had a higher 5 year relative survival compared with those who received surgery alone, surgery and radiotherapy, and other combinations of treatment.

\section{Limitations of the study}

Values for the risk of mesothelioma by primary care trust of residence must be interpreted with caution. Residential areas of high incidence do not necessarily indicate areas where exposure occurred. Lack of information on migration patterns and

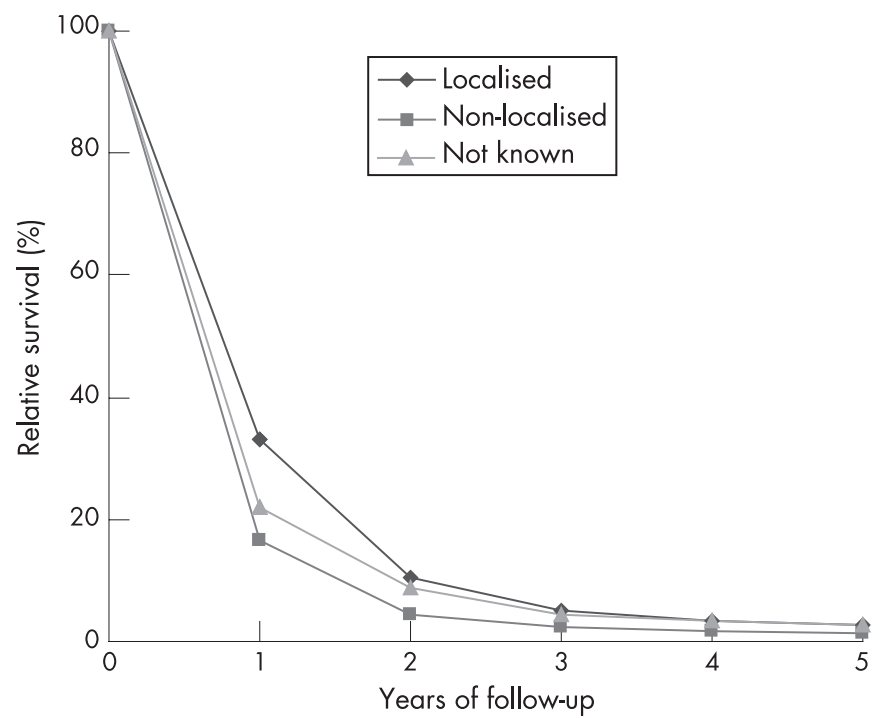

Figure 7 Relative survival of mesothelioma in men by disease stage, South East England (1998-2002). occupation of these patients meant that we could not explore the specific reasons for high incidence in some areas. However, it is known that those who have worked in the shipbuilding, locomotive and insulation industries with past asbestos use are at higher risk of mesothelioma. Regarding information on treatment, cancer registration data include records of treatment received within 6 months of diagnosis. Patients classified under "no treatment recorded" may therefore have received treatment at a later date. The variation in the treatment received across the cancer networks may to a degree reflect the variation in ascertainment of treatment details as well as variation in clinical practice for the treatment of this disease. Patients notified to the cancer registration system by death certificate only have been excluded from the survival analysis, and in some areas with a higher proportion of these notifications, the relative survival may be artificially inflated.

\section{Implications}

Clinical practice

Mesothelioma is predominantly a disease of men. The incidence in women remained much lower and was relatively stable over the study period. Women have been less often directly exposed to asbestos in the occupational setting but they are at risk when members of their family worked with asbestos. ${ }^{13}$

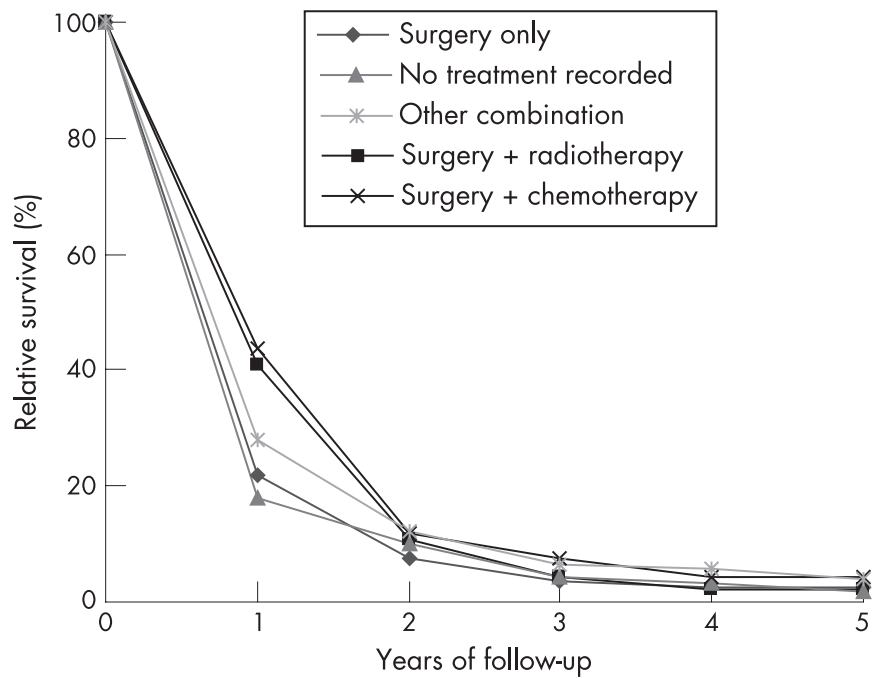

Figure 8 Relative survival of mesothelioma in men by treatment type, South East England (1998-2002). 
There is currently no evidence to indicate that a specific treatment leads to improved survival in mesothelioma patients. ${ }^{14}$ Our study did not show any major differences in survival across the cancer networks and provides no further advice about the choice of treatment that could lead to improved survival. Other studies have found that longer survival is associated with small epithelial type, node negative pleural mesotheliomas, aggressive surgery combined with adjuvant chemotherapy and or radiotherapy, and early stage disease, good performance status and good haematological status. ${ }^{1415}$ Extrapleural pneumonectomy (removal of the pleura with the lung, pericardium and diaphragm) within multimodal treatment may be considered for patients with early stage disease, although there are no randomised trials of this treatment and complications have been reported. ${ }^{16}$ Studies have shown that the most common symptoms experienced by patients are pain, breathlessness, fatigue, appetite loss, insomnia and cough. ${ }^{17}{ }^{18}$ Chemotherapy may improve some of these symptoms although not sufficiently to improve role function and at the expense of side effects. There is, however, evidence that some of these symptoms can be targeted for control using other methods. A multicentre randomised controlled trial of a nursing intervention to provide breathing control, pacing of activity, relaxation techniques and psychosocial support for patients with lung cancer or mesothelioma found improved breathlessness, performance status and emotional states in those receiving the intervention compared with controls. ${ }^{19}$ Despite some evidence of variation in treatment between cancer networks, there was no corresponding variation in survival.

Clearly an important focus must be to ensure high quality palliative care for these patients. Established quality of life measures are now being tested on patients with mesothelioma and applied within clinical trials to determine whether treatment has an impact on health related quality of life and other palliative outcomes.

\section{Health policy and research}

The Chief Medical Officer recently highlighted the future epidemic of this disease in his recent report for England and Wales on the state of public health. ${ }^{20}$ National guidelines specific to this disease would be useful to ensure that patients are provided with appropriate services and information relating to their illness. The important roles of carers, nurses and families who help these patients cope with the disease cannot be overlooked either. The guidelines would also need to assist patients deal with the legal implications should they decide to seek compensation.

Cancer networks, particularly those with primary care trusts that have high incidence, need to be aware of this disease and ensure that services are in place to assist these patients. This includes directing patients to voluntary organisations such as the British Lung Foundation ${ }^{21}$ and the Roy Castle Lung Cancer Foundation $^{22}$ that are able to provide non-clinical support. The primary care trusts in their commissioning role need to ensure that sufficient funds are allocated to provide services for mesothelioma patients, particularly when the incidence of mesothelioma is expected to increase in the near future. Patients who are willing should be encouraged to participate in clinical trials in order to increase the understanding of this disease. Not only should we be interested in the biological aspects of this disease but we should also learn about the emotional aspects experienced by the patients, their families and carers.

The challenges ahead include raising awareness of this disease among health professionals, diagnosing patients quickly, providing coordinated cancer services to alleviate their symptoms, support them and their families, and continuous research into the treatment of this disease. Evidence on effective treatment and palliative care is urgently required. A national strategy for these patients similar to other patient groups would be one way forward, and a Department of Health framework for the development of service guidance for these patients is now in discussion..$^{23}$

Acknowledgements: We thank Professor Tom Treasure for his comments on an earlier version of this manuscript.

Competing interests: None.

\section{REFERENCES}

1. Jones B, Thomas P. Incidence of mesothelioma in Britain. Lancet 1986;1:1275.

2. Bianchi C, Giarelli L, Grandi G, et al. Latency periods in asbestos-related mesothelioma of the pleura. Eur J Cancer Prev 1997;6:162-6.

3. Peto J, Decarli A, La VC, et al. The European mesothelioma epidemic. Br J Cancer 1999;79:666-72.

4. Hemminki K, Li X. Mesothelioma incidence seems to have leveled off in Sweden. Int J Cancer 2003;103:145-6.

5. Pelucchi C, Malvezzi M, La VC, et al. The mesothelioma epidemic in Western Europe: an update. Br J Cancer 2004;90:1022-4.

6. Hodgson JT, McElvenny DM, Darnton AJ, et al. The expected burden of mesothelioma mortality in Great Britain from 2002 to 2050. Br J Cancer 2005:92:587-93.

7. McElvenny DM, Darnton AJ, Price MJ, et al. Mesothelioma mortality in Great Britain from 1968 to 2001. Occup Med (Lond) 2005;55:79-87.

8. O'Reilly D, Reid J, Middleton R, et al. Asbestos related mortality in Northern Ireland: 1985-1994. J Public Health Med 1999;21:95-101.

9. Treasure T, Sedrakyan A. Pleural mesothelioma: little evidence, still time to do trials. Lancet 2004;364:1183-5.

10. British Thoracic Society Standards of Care Committee. Statement on malignant mesothelioma in the United Kingdom. Thorax 2001;56:250-65.

11. Elliot P, Cuzick J, English D, et al. Geographical and environmental epidemiology: methods for small area studies. Practical approaches to disease mapping. Oxford: University Press/World Health Organization, 1996.

12. Brenner $\mathbf{H}$, Gefeller 0 . An alternative approach to monitoring cancer patient survival. Cancer 1996; 18:2004-10.

13. Walach N, Novikov I, Milievskaya I, et al. Cancer among spouses: review of 195 couples. Cancer 1998;82:180-5.

14. Maziak DE, Gagliardi A, Haynes AE, et al. Surgical management of malignant pleural mesothelioma: a systematic review and evidence summary. Lung Cancer 2005;48:157-69.

15. Steele JP, Klabatsa A, Fennell DA, et al. Prognostic factors in mesothelioma. Lung Cancer 2005;49(Suppl 1):S49-52.

16. Treasure $\mathbf{T}$, Waller $\mathrm{D}$, Swift $\mathrm{S}$, et al. Radical surgery for mesothelioma. BMJ 2004;328:237-8.

17. Hollen PJ, Gralla RJ, Liepa AM, et al. Adapting the Lung Cancer Symptom Scale (LCSS) to mesothelioma: using the LCSS-Meso conceptual model for validation. Cancer 2004;101:587-95.

18. Nowak AK, Stockler MR, Byrne MJ. Assessing quality of life during chemotherapy for pleural mesothelioma: feasibility, validity, and results of using the European Organization for Research and Treatment of Cancer Core Quality of Life Questionnaire and Lung Cancer Module. J Clin Oncol 2004;22:3172-80.

19. Bredin M, Corner J, Krishnasamy M, et al. Multicentre randomised controlled trial of nursing intervention for breathlessness in patients with lung cancer. BMJ 1999;318:901-4.

20. Chief Medical Officer. On the state of public health: annual report of the Chief Medical Officer of the Department of Health. London: Department of Health, 2005.

21. British Lung Foundation. http://www.lunguk.org (accessed 29 November 2007).

22. Roy Castle Lung Cancer Foundation. http://www.roycastle.org (accessed 29 November 2007).

23. Department of Health. Mesothelioma Framework. http://www.dh.gov.uk (accessed 29 November 2007). 